\title{
A load of small RNAs in the sperm - how many bits of hereditary information?
}

\author{
Jafar Kiani ${ }^{1,2,3}$, Minoo Rassoulzadegan ${ }^{1,2,3}$
}

${ }^{1}$ Inserm, U1091, Nice, F-06108, France; ${ }^{2}$ CNRS, UMR7277, F-06108, France; ${ }^{3}$ University of Nice-Sophia Antipolis, UFR Sci-

ences, Nice, F-06108, France

Cell Research (2013) 23:18-19. doi:10.1038/cr.2012.181; published online 25 December 2012

Transcriptionally silent sperm contains a variety of RNA fragments of both coding and non-coding transcripts. A recent article by Peng and colleagues reveals several new families of small RNAs enriched in sperm, which are derived from the same locus as tRNAs. The finding of these short fragments of tRNA in the sperm raises once again the question of the possible function(s) of such a miniaturized form of information carried by the spermatozoon.

Germ cells prepare the transmission of information to the next generation. The logic of Mendelian heredity accounts for a large fraction of it, but over the past few years several independent investigations lead us to also consider other forms of heredity. One of the possibilities is a non-Mendelian form of transmission of epigenetic informationregulatory mechanisms that expression of genes is either silenced or activated in a manner stable enough to be maintained throughout cellular divisions ('epigenetic memory') and even transferred to the following generations ('epigenetic heredity'). What drives such alternative epigenetic phenomena, what targets genetic loci in a sequence-specific manner, and for paternal heredity, what regulatory components are comprised in

Correspondence: Minoo Rassoulzadegan

E-mail:minoo@unice.fr the minimal cellular structure of sperm? Various candidates are considered, such as modulations of DNA methylation and covalent modifications of nucleosomal histones in chromatin. However, RNA molecules are now also considered as key vectors of epigenetic variations with recent development of experimental assays in organisms ranging from the worm to the fly and the mouse. RNAs would obviously be the best candidates to act by sequence recognition as guides of complexes that would include protein and other components of silencing/activating systems. Hence, the development of research programs to investigate RNA molecules in the most detailed manner, i.e., by deep sequencing the RNA content of the germ cell, with a specific interest in sperm, a transcriptionally silent cell with a limited amount of RNA to sequence, could hold the keys to biological effects.

Mature mammalian sperm is produced after complex steps of differentiation of the cytoplasm (formation of the chromatoid body) and the nucleus (compacted chromatin structure). Only 10-20 fg of RNA are maintained in a mature sperm cell, with a profile distinct from somatic cells because of the absence of the major components of the ribosome. Although part of the sperm RNA is made of fragments of coding transcripts, several exploratory analyses identified a variety of non-coding RNAs (ncRNAs). Krawetz and his colleagues [1] reported that spermatozoa contain an abundant population of ncRNA - about 24000 estimated in an individual sperm. This small RNA fraction includes multiple known classes of ncRNAs: repeat-associated small RNAs, miRNAs, siRNAs, and piRNAs. Approximately $20 \%$ of the miRNAs are introduced into the oocyte during fertilization [2]. It was shown that miR34c is a sperm-borne microRNA [3]. Two examples of siRNAs were found in sperm, IGF2 receptor antisense and antisense sequence for the Dickkopf-2 gene [4]. piRNAs, initially discovered in differentiating germ cells, are also found in sperm. Recently, Kawano et al. [5] reported another class of small RNAs in low copy number derived from a piRNA locus. By high-throughput sequencing of small-RNAs ( $<40 \mathrm{bp})$ in the sperm, Peng and colleagues [6] now show a marked enrichment of tRNA fragments of 30-34 nt in length in the sperm, which make up to $67 \%$ of total sperm small RNA reads. They pointed out that these RNAs are matched to the $5^{\prime}$ halves of tRNAs, hence they are designated as mature sperm-enriched tRNA-derived small RNAs or mse-tsRNA, which are generated from mature tRNAs through cleavage at specific site within the anti-codon loops of a majority of glu- and gly-tRNAs. Intriguingly, these mse-tsRNAs are highly enriched in mature sperm head and oocytes, but their numbers drop dramatically after 
fertilization.

The search is now open to define possible functions of these mse-tsRNAs in fertilization, in zygotes or in embryonic development, which may eventually undergo variations constitutive of a mode of inheritance. tRNAs are abundant and functionally important noncoding RNAs with highly conserved and extensive posttranscriptional processing. Maturation of tRNA generates several byproduct species, including tRNA fragments derived from tRNA 3'-trailer processed by tRNase $Z$ or tRNA 5'-leader by RNase P. A parallel has been drawn with a different set of RNAi machinery enzymes [7]. A functional difference independent of protein synthesis is suggested with a novel role in modulation of gene expression. Upon environmental changes like stress conditions, starvation, heat shock or oxidative stress, such tRNA fragments dramatically increase in frequency. It was shown that these cleaved $5^{\prime}$ halftRNAs can suppress protein synthesis independent of eIF $2 \alpha$ in the transfected cell [8]. In the same study, suppression of protein synthesis was associated with displacement of eIF4G/A from mRNAs by tRNA fragments. Moreover, these fragments can induce formation of stress granules and cytoplasmic foci with untranslated mRNPs [9]. Elbarbary and coworkers [10] have studied gene expression modulated by human 5'-half-tRNA coupled with tRNase $Z^{\mathrm{L}}$ or tRNase Zl activity. 5'-half-tRNA acts as a small guide RNA (sgRNA) for tRNase $\mathrm{Z}^{\mathrm{L}}$, and efficiently leads to the suppression of gene expression of a specific mRNA containing complemen- tary binding sequence of sgRNA. They suggest that tRNase $Z^{\mathrm{L}}$ guided by tRNA halves can play a role in p53 signaling pathway and apoptosis.

The discovery of an enrichment of 5 '-half tRNA fragments in sperm now raises the question of the biological meaning of what we read. What is the function of these small RNAs if any? The next step, more challenging, is to find a biological test to support the sequencing data. One more and more obvious possibility is that epigenetic modifications are induced by such RNA molecules, which, is now being tested experimentally in multiple organisms, from worms and Drosophila to mammals [11-15]. The findings of Peng and colleagues add hope to a growing field to understand how the maintenance of such large amounts of fragmented tRNAs in sperm functionally contributes to the non-Mendelian transmission of heritable traits.

\section{References}

1 Krawetz SA, Kruger A, Lalancette C, et al. A survey of small RNAs in human sperm. Hum Reprod 2011; 26:34013412.

2 Amanai M, Brahmajosyula M, Perry AC. A restricted role for sperm-borne microRNAs in mammalian fertilization. Biol Reprod 2006; 75:877-884.

3 Liu WM, Pang RT, Chiu PC, et al. Sperm-borne microRNA-34c is required for the first cleavage division in mouse. Proc Natl Acad Sci USA 2012; 109:490-494.

4 Krawetz SA. Paternal contribution: new insights and future challenges. $\mathrm{Nat}$ Rev Genet 2005; 6:633-642.

5 Kawano M, Kawaji H, Grandjean V,
Kiani J, Rassoulzadegan M. Novel small noncoding RNAs in mouse spermatozoa, zygotes and early embryos. PLoS One 2012; 7:e44542.

6 Peng H, Shi J, Zhang Y, et al. A novel class of tRNA-derived small RNAs extremely enriched in mature mouse sperm. Cell Res 2012; 22:1609-1612.

7 Sobala A, Hutvagner G. Transfer RNAderived fragments: origins, processing, and functions. Wiley Interdiscip Rev RNA 2011; 2: 853-862.

8 Ivanov P, Emara MM, Villen J, Gygi SP, Anderson P. Angiogenin-induced tRNA fragments inhibit translation initiation. Mol Cell 2011; 43:613-623.

9 Emara MM, Ivanov P, Hickman T, et al. Angiogenin-induced tRNA-derived stress-induced RNAs promote stressinduced stress granule assembly. J Biol Chem 2010; 285:10959-10968.

10 Elbarbary RA, Takaku H, Uchiumi $\mathrm{N}$, et al. Modulation of gene expression by human cytosolic tRNase $Z^{\mathrm{L}}$ through 5'-half-tRNA. PLoS One 2009; 4:e5908.

11 Wagner KD, Wagner N, Ghanbarian H, et al. RNA induction and inheritance of epigenetic cardiac hypertrophy in the mouse. Dev Cell 2008; 14:962-969.

12 Rassoulzadegan M, Grandjean V, Gounon P, et al. RNA-mediated nonmendelian inheritance of an epigenetic change in the mouse. Nature 2006; 441:469-474.

13 de Vanssay A, Bouge AL, Boivin A, et al. Paramutation in Drosophila linked to emergence of a piRNA-producing locus. Nature 2012; 490:112-115.

14 Chandler VL. Paramutation: RNA-mediated instructions passed across generations. Cell 2007; 23:641-645.

15 Ashe A, Sapetschnig A, Weick EM, et al. piRNAs can trigger a multigenerational epigenetic memory in the germline of C. elegans. Cell 2012; 150:8899. 